\title{
CARACTERIZACIÓN DE QUESO CHANCO ENRIQUECIDO CON SUERO LÁCTEO EN POLVO
}

\section{CHARACTERIZATION OF CHANCO CHEESE ENRICHED WITH POWDER MILKWHEY}

\author{
Margarita Arteaga M. (1), Luz Haydeé Molina C. (2), Manuel Pinto C. (2) Carmen Brito C. (2). \\ (1) Programa de Ingeniería de Alimentos. Universidad de Córdoba. Montería. Colombia. \\ (2) Instituto de Ciencia y Tecnología de los Alimentos. Universidad Austral de Chile. Valdivia, Chile.
}

\begin{abstract}
The influence by the addition of whey proteins on the biochemical changes in Chanco cheese ripening was evaluated. 4 treatments were set up: TC(control), and T1, T2 and T3 adding milk whey powder at 2, 4, $6 \%(\mathrm{~m} / \mathrm{m})$ respectively using a factorial design $3 \times 4$ (4 treatments by 3 repetitions). In pH control, humidity, fat, total solids, proteins, ashes and salt at 0, 14, 28 and 42 days, standardized methods were used: IDF-FIL, AOAC and Chilean guidelines. Color by colorimeter Color test LFM1, water activity by the LUF analyzer and by the formula. In the sensorial evaluation the test of Multiple Comparison (4 attributes) was used. The results showed that glycolysis increased during ripening in treatments with whey. Moisture, fat and ashes increased at $T 2 \& \mathrm{~T} 3$, and water activity decreased slowly in cheeses. Control and T1 in texture profile were statistically equal. It is concluded that whey addition to Chanco cheese it is feasible but limited.
\end{abstract}

Key words: Chanco cheese, milk whey powder.

Este trabajo fue recibido el 16 de Septiembre de 2008 y aceptado para ser publicado el 20 de Enero de 2009.

\section{INTRODUCCIÓN}

En la concentración de la leche durante el procesamiento del queso, se libera lactosuero que corresponde a cerca del $83 \%$ del volumen de leche utilizada como materia prima, el cual es exudado desde el interior de la cuajada de queso. Contiene alrededor del $50 \%$ de la concentración de los sólidos de la leche y particularmente por sus proteínas séricas, que son de muy buena calidad debido a que aportan aminoácidos esenciales y poseen un alto coeficiente de absorción, es considerado un producto de alto valor nutritivo. Además, contiene lactosa y un perfil de minerales donde se destaca la presencia de potasio, lo que favorece la eliminación de líquidos y toxinas del organismo, dispone también de calcio, fósforo y magnesio, y oligoelementos como zinc, hierro y cobre, formando sales de gran biodisponibilidad para el organismo. Adicionalmente, posee vitaminas A, C, D, E y del complejo B, así como ácido orótico, que es fundamental para la absorción de las minerales, y ácido láctico que ayuda a mejorar el proceso de respiración celular, junto con un contenido muy bajo en grasas y calorías $(1,2)$. Consecuentemente el suero lácteo representa un material de alto interés para usar en consumo humano.

Paralelamente, desde hace algunos años se está estudiando la relación entre el incremento en la ingesta de calcio y del impactante rol que éste puede jugar en la regulación del metabolismo energético y modulación del riesgo de sobrepeso y obesidad en las personas. Las investigaciones aún no entregan resultados totalmente concluyentes, sin embargo diversos autores señalan que el consumo de lácteos reducidos en grasa y de alto nivel de calcio podría contribuir a reducir el problema de obesidad cada vez mayor en el mundo (3 - 6). Lo anterior podría también aplicarse a la realidad chilena, frente a los grandes y actuales problemas de sobrepeso y obesidad que acontece en esta población, ampliamente sustentado en artículos científicos (7 - 11).

Para la industria alimentaria, el suero constituye 
una fuente económica de proteínas, que otorga múltiples propiedades de aplicación en una amplia gama de alimentos, debido a sus diversas capacidades funcionales. Los productos de suero mejoran la textura, realzan el sabor y color, presentan características de emulsificantes y estabilizantes, mejoran las propiedades de flujo, entre varias otras propiedades tecnofuncionales, lo que posibilita incrementar con éste, la calidad de muchos productos alimenticios $(12,13)$.

Por tal motivo, progresivamente el suero se ha ido industrializando y utilizando en mayor volumen en la elaboración de productos para alimentación humana, además del tradicional uso en alimentación animal. Así, su uso en bebidas fermentadas, productos de panadería, repostería, dulces, además de productos químicos y farmacéuticos como ácido láctico, alcohol etílico, etc. $(14,15)$.

Otra forma de utilizar este importante producto, es agregarlo para enriquecer los sólidos de la leche, por ejemplo para incrementar a la vez, el rendimiento y el valor nutricional de los quesos madurados. No obstante, tal adición podría afectar el proceso y/o algunas de las características típicas de las variedades de queso, particularmente podría verse afectada la maduración del producto, etapa en que ocurren los cambios bioquímicos más relevantes que originan las particulares características sensoriales típicas de cada variedad como consistencia, color, textura, sabor y olor $(16,17)$.

La adición de proteínas del suero a los quesos aumenta la cantidad de agua ligada dado que se forman complejos entre las proteínas desnaturalizadas del suero y la caseína de la leche, por lo que su disponibilidad como solvente es probablemente menor. Este comportamiento influye críticamente en las reacciones bioquímicas de maduración del queso, lo cual junto con el incremento del contenido de lactosa, podría reducir la calidad del producto al elevar la acidez de éste afectando negativamente su consistencia y textura $(16,18)$.

El Chanco, es un queso madurado, de origen chileno y preferido del consumidor nacional, que se elabora con leche pasteurizada de vaca. Se obtiene por coagulación enzimática coadyuvada por la acidez desarrollada por cultivos lácticos puros. Su maduración debe ser por lo menos de 21 días $(19,20)$.

A la vista de las importantes características nutritivo dietéticas y tecno - funcionales del suero lácteo, la presente investigación tuvo por objetivo comparar el curso de la maduración y calidad del queso Chanco elaborado sin y con diversos niveles de enriquecimiento de la leche con suero. Para ello se comparó: a) las características físicas y químicas de los quesos producidos, a los 0 , 14, 28 y 42 días de maduración, b) las características sensoriales de los quesos madurados (28 y 42 días), se estableció la relación entre c) la actividad de agua (aw) medida instrumentalmente y por fórmula y d) el color, medido por método sensorial e instrumental, a los 28 y 42 días de maduración.

\section{MATERIAL Y MÉTODO}

\section{Materiales}

Se dispuso de muestras de queso Chanco con diferentes niveles de incorporación de suero en polvo a la leche utilizada para su elaboración $(2,4$ y $6 \%$ ) correspondientes a: T1, T2 y T3, respectivamente y muestras de queso Chanco sin adición de suero (TC).

Los análisis correspondieron a: $\mathrm{pH}$, humedad, materia grasa, sólidos totales, proteína, cenizas y sal, realizados con el objetivo de identificar la variedad específica; para lo cual se utilizaron métodos de análisis estandarizados, Normas IDF- FIL, AOAC y Normas Chilenas (INN). (21-26).

Para determinar el color, se usó el colorímetro Color Tester LFM 1. Dr. Lange. DIN 5033 (27). La aw se midió por el analizador de aw LUF, modelo 5803. (28) y en el cálculo de aw se usó la fórmula (29), que se presenta a continuación:

aw: 1,0234 - 0,0070 [Contenido de cenizas (g)/ $100 \mathrm{~g}$ agua del queso]

\section{Diseño experimental}

Se planteó un diseño factorial de 3 x 4 considerando 4 tratamientos y 3 repeticiones y el efecto del tiempo de maduración $(0,14,28$ y 42 días). Los análisis se realizaron por duplicado.

La evaluación sensorial de los quesos se realizó por el método de Comparación Múltiple, test de tipo diferencial de respuesta objetiva, en el cual 7 panelistas entrenados evaluaron los atributos de color, aroma y gusto, textura y aceptación general del producto, mediante escala de intensidad de 10 puntos.

\section{Análisis estadístico}

A los resultados de los análisis físicos y químicos se les aplicó el Test de homoscedasticidad de varianzas, Análisis de varianza (ANOVA) de una vía y análisis de comparación múltiple (Test de Tukey). Los resultados sensoriales, fueron analizados mediante Test de concordancia Prueba de Kruskal-Wallis, Análisis de comparación múltiple y se determinó un perfil de los quesos por tratamiento (Nota modal). 


\section{RESULTADOS Y DISCUSIÓN}

\section{Evolución del pH}

En la figura 1, se observa que a inicios de maduración, únicamente el Tratamiento Control (TC) está acorde con lo señalado por la literatura $(30,31)$, en que se indica que el $\mathrm{pH}$ de los quesos semiduros y el Chanco en particular, al inicio de la maduración debería estar entre 5,2 y 5,25. Los restantes tratamientos están por sobre estos valores, lo cual es atribuible al efecto de la capacidad tampón que tienen estas cuajadas por el agregado de suero, particularmente por su contenido proteico (proteínas séricas) lo que, junto con el mayor nivel de lactosa, puede variar el perfil normal de la fermentación haciéndola más lenta al inicio pero más intensa al final.

Por lo anterior, la glicólisis, que normalmente debería llegar a su término dentro de las dos primeras semanas de maduración, a diferencia del tratamiento control, en los tratamientos con suero continúa durante toda la maduración, esto se refleja en el descenso sostenido del $\mathrm{pH}$ lo que se manifiesta en una curva con pendiente inversa opuesta a la presentada por el control durante esta etapa. Lo anterior afectó la calidad sensorial del producto, que los panelistas detectaron como un sabor ácido.

El comportamiento del $\mathrm{pH}$ es sustentado en el alto porcentaje de lactosa que presenta el suero en polvo (61-75\%) (16), la cual es susceptible de degradar, así, al aumentar el porcentaje de suero de los tratamientos, se incrementa la discrepancia del $\mathrm{pH}$ entre el día 0 y 42 de maduración. Como resultado, la incorporación de 2, 4 y $6 \%$ de suero en polvo, según el tratamiento, provoca un diferencial de 0,$21 ; 0,38$ y 0,44 en el $\mathrm{pH}$ respectivamente, lo que confirma que cuanto mayor es el contenido de lactosa, más rápidamente se desarrollan los microorganismos en el mismo intervalo de tiempo.

Pese a lo anterior, con el tiempo de maduración los comportamientos disminuyen sus diferencias y a los 28 días de maduración, los $\mathrm{pH}$ de los 4 tratamientos son semejantes y se encuentran dentro del rango $5.2-5.4$, que establece para el Chanco la Norma NCh 2090 Of. 1999. (19).

\section{Evolución de humedad y sólidos totales}

Como se aprecia en la tabla 1, los valores de sólidos totales (ST), en todos los tratamientos y fechas evaluadas se encuentran dentro del rango exigido por la norma chilena de la variedad, correspondiente a 52 a $56 \%(\mathrm{~m} / \mathrm{m})$.

ECK, en 1990 (33), afirmó que en variedades de queso sin protección, es decir quesos con cáscara, como es el caso del Chanco, durante su maduración pierde humedad siempre y de forma progresiva, hasta alcanzar el equilibrio de la aw de su superficie con la humedad relativa del ambiente. Las pérdidas de humedad a los días 28 y 42 estuvieron entre 3,16 y 4,17 y entre 4.0 y 5.26

\section{FIGURA 1}

Evolución del pH en la maduración de queso Chanco con y sin suero

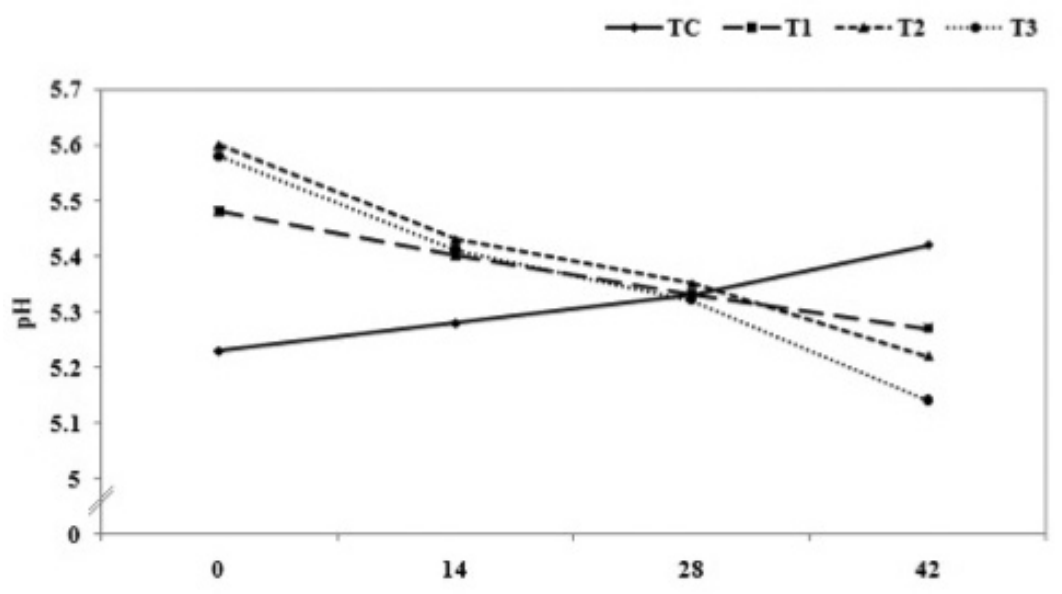

Tiempo de maduración (días) 
$\%(\mathrm{~m} / \mathrm{m})$ respectivamente, todas dentro de normales técnicas respecto a la variedad y de las condiciones de maduración (humedad relativa y temperatura).

\section{Evolución de la actividad de agua (aw)}

En la figura 2, se puede apreciar que en general a medida que transcurre la etapa de maduración acontece, en todos los tratamientos, un descenso en la aw, correspondiente a 0,$018 ; 0,035 ; 0,027$ y 0,035 unidades, para los tratamientos TC, 1, 2, 3 respectivamente, siendo levemente menor en el tratamiento control frente a los obtenidos en los quesos con adición de suero.

La aw describe la proporción de agua libre que contiene el queso, por tanto el agua disponible para las reacciones enzimáticas propias de maduración, como también de aquellas que provocan riesgos de deterioro del producto. Así lo confirman Esteban \& Marcos y Asmundson et al. $(29,34)$, al afirmar que la aw del queso disminuye durante la elaboración, el salado y la maduración, como resultado de la concentración de los sólidos y además, por la producción de compuestos nitrogenados no proteicos de bajo peso molecular y de solutos solubles derivados de los procesos de glicólisis, proteólisis y lipólisis.

El análisis de varianza individual del aw, no muestra diferencias estadísticamente significativas a un nivel de confianza del $95 \%(\mathrm{~m} / \mathrm{m})$, entre los cuatro tratamientos evaluados durante la maduración del queso Chanco.

Los resultados de aw obtenidos en el estudio por ambos métodos, presentan el mismo comportamiento (figura 2), aw disminuye a medida que avanza el proceso de maduración, comportamiento paralelo a la humedad, no existiendo diferencias significativas entre tratamientos, cuyo coeficiente de correlación entre métodos (r: 0,81), es altamente significativo a un nivel de confianza del $95 \%$, por lo que se concluye que el cálculo por fórmula resulta adecuado para usar en producciones de queso Chanco.

\section{Contenido de cloruro y cenizas}

En la tabla 1, se observa que el tratamiento control presenta menor contenido de cloruros (sal) y cenizas, que el resto de los tratamientos.

El suero contiene minerales de calcio, fósforo, sodio, potasio y magnesio, que constituyen las sales y parte

\section{TABLA 1}

\section{Evolución de los parámetros composicionales en maduración del Chanco (g/100 g).*}

\section{Tiempo de}

maduración

Parámetros

químicos

Sólidos totales

Cloruros

Cenizas

Proteína

Grasa

\section{Tiempo de}

maduración

Parámetros

químicos

Sólidos totales

Cloruros

Cenizas

Proteína

Grasa

\section{0 días}

\section{Tratamientos}

C

53,37 a

$1,06 \mathrm{a}$

$3,05 \mathrm{a}$

22,03 a

26,80 a
1

2

3

$52,54 \mathrm{a}$

$1,28 \mathrm{ab}$

3,43 a

51,37 a

$1,46 \mathrm{~b}$

$20,56 a b$

3,69 a

27,13 a

$18,71 \mathrm{bc}$

$27,47 \mathrm{ab}$

C

51,31 a

54,30 a

1,26 a

3,61 a

$17,55 \mathrm{c}$

3,45 a

22,99 a

$29,15 \mathrm{~b}$
14 días

\section{Tratamientos}

1

2

3

* Promedio de tres repeticiones, análisis en duplicado.

Letras distintas indican diferencia significativa entre tratamientos, a un nivel del $95 \%$ de confianza. 
de las cenizas de la leche y del suero, el porcentaje de cenizas en suero en polvo comprende un rango del 7 al $14 \%(\mathrm{~m} / \mathrm{m})(35,36)$.

Los $\mathrm{pH}$ de los tratamientos 1,2 y 3 , obtenidos durante el proceso de elaboración de los quesos son más altos que el obtenido en el tratamiento control, por lo que en este último se esperaría una mayor desmineralización y por ende un menor porcentaje de cenizas en la masa del queso.

A medida que aumenta el porcentaje de "humedad en el queso desgrasado", en los diversos tratamientos, se observó un incremento paulatino en el porcentaje de sal y de cenizas, lo que se evidencia claramente en T3. Brito et al., (37), encontraron en Chanco típico, valores de $1,28 \% \pm 0,14$, y Molina et al., (39), entre 1,42 y $1,83 \%$, para el contenido de sal en base total, el rango más bajo lo presenta el control (1.41 g de sal por $100 \mathrm{~g}$ de queso), siendo estadísticamente diferente a los otros tratamientos, $\mathrm{T} 1$ y T2 poseen niveles intermedios (1.64 y $1.79 \mathrm{~g} / 100 \mathrm{~g}$ ) siendo T3 estadísticamente diferente y el más alto con $1.93(\mathrm{~g} / 100 \mathrm{~g})$ a los 28 días de maduración, no obstante, a los 42 días los tres tratamientos con suero aparecen diferentes estadísticamente al control y ligeramente por sobre el máximo indicado por los autores señalados, lo que también, en la evaluación sensorial, fue percibido como productos ligeramente más salados. La norma chilena no establece especificaciones sobre el contenido de sal.

\section{Contenido de materia grasa}

Todos los tratamientos se encuentran dentro del rango establecido por la Norma Chilena Oficial NCH 2090 (19), la que estipula un mínimo para materia grasa en base total del $25 \%(\mathrm{~m} / \mathrm{m})$ y para materia grasa en extracto seco del $45 \%(\mathrm{~m} / \mathrm{m})$, en el momento de la comercialización del queso. En la tabla 1, se observa un ascenso relativo en la expresión de la materia grasa durante la maduración, que va para el tratamiento control, entre $26.80 \mathrm{~g} / 100 \mathrm{~g}$, hasta 27,87 y $28,97 \mathrm{~g} / 100 \mathrm{~g}$ a los 28 y 42 días respectivamente $\mathrm{y}$, hasta 30,53 y $31,68 \mathrm{~g} / 100 \mathrm{~g}$ a los 28 y 42 días, respectivamente en T3, sin diferencias estadísticamente significativas entre tratamientos, lo cual sólo de debe a la concentración de sólidos por la pérdida de humedad de los quesos durante maduración.

\section{Contenido de proteína}

La proteína presentó en los tratamientos, un contenido con valores que van entre 18,89 y $23,50 \mathrm{~g} / 100 \mathrm{~g}$ a fines de la maduración y de 19,95 y 23,99 g/100g a los 42 días. El balance de proteínas realizado en la elaboración del queso, muestra que las pérdidas de nitrógeno en el suero fueron desde $0,89 \mathrm{~g} / 100 \mathrm{~g}$ para el tratamiento control, y hasta $1,39 \mathrm{~g} / 100 \mathrm{~g}$ en el resto de los tratamientos, lo que da cuenta de la disminución de la proteína obtenida en los quesos de los tratamientos con suero frente al control (tabla 1).

Según lo indicado por la literatura, al parecer, las

\section{FIGURA 2}

\section{Evolución de la aw (teórica e instrumental), en la maduración de queso Chanco con y sin suero}

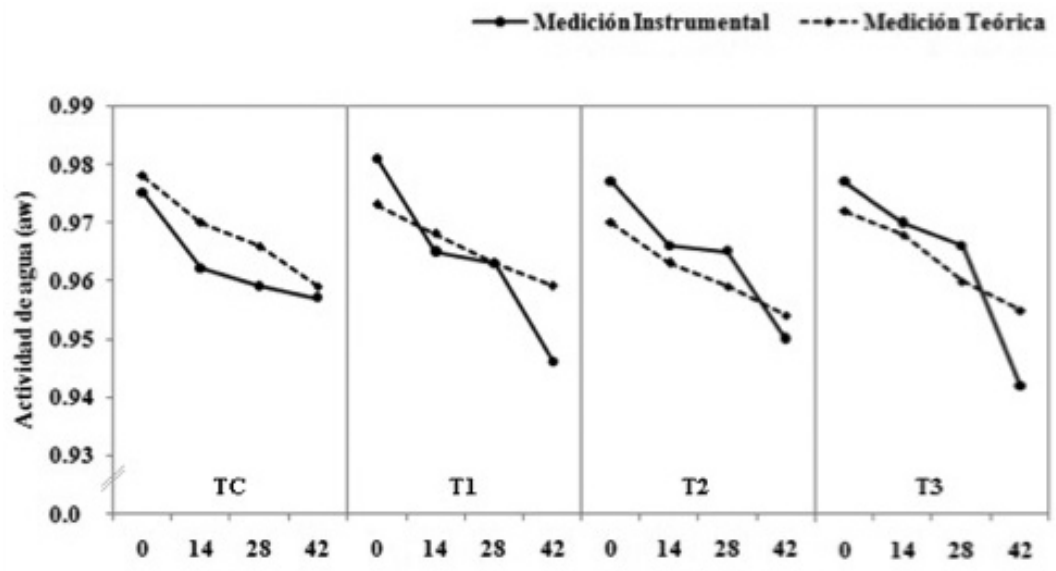

Tiempo de maduración (dias) 
proteínas séricas incorporadas presentan un comportamiento similar a la grasa frente a la red caseínica, actuando sólo como un material inerte y de relleno en ésta, por lo que ambas (grasa y proteínas séricas), competirían por los mismos espacios en la red estructural de la cuajada, donde serían simplemente poros de la red de caseína (17), lo que probablemente afecta negativamente la retención de altas concentraciones de proteína sérica en la masa de queso. Los resultados obtenidos en este estudio son coincidentes con lo reportado en literatura al elaborar Mozzarella con adición de suero, donde se encontró que las pérdidas de proteínas en el proceso eran un $51 \%$ superior el nivel de proteína perdida en los procesos sin adición de suero (38).

\section{Características sensoriales}

Color: Según la norma Chilena 2090 (19), el color externo del queso Chanco debe ser amarillo pálido y la masa interna blanca cremosa o amarillo pálido, homogéneo.

Los puntajes asignados al color en los quesos correspondientes a T3 son los más bajos (figura 3) que, frente al concepto de calidad quedan "bajo lo normal" ( 6,0 y 6,1 ptos), T2 se encuentra en este mismo concepto, aunque con valores ligeramente superiores $(7,6$ y 7,0), los panelistas señalan como defecto, un color heterogéneo (moteado) y muy pálido. Los tratamientos control y T1 no presentan diferencias significativas, obteniendo los mayores puntajes $(8.7-9,0$ y 8,0 - 8,0), para 28 y 42 días respectivamente, lo que corresponde a un "color normal".

Los defectos observados en T2 y T3 se podrían atribuir a la continuidad de acidificación durante maduración, lo que retrasa los procesos bioquímicos normales que originan los cambios requeridos en textura y color de la masa del queso. Además, la incorporación de suero originó algunos inconvenientes en el proceso, particularmente en la obtención de la cuajada por la débil consistencia del gel obtenido durante la etapa de coagulación, ocasionando dificultad en la operación de corte y agitación de la cuajada, lo que produjo un grano heterogéneo, que probablemente ocasionó desuniformidad de color al producirse vetas y manchas blancas en la masa del queso.

El test de Kruskal - Wallis confirma que para el atributo color, medido sensorialmente a los 28 días de maduración hay diferencia entre el grupo TC - T1, con respecto a los tratamientos $\mathrm{T} 2$ y $\mathrm{T} 3$.

En la figura 3 se aprecian los valores de las coordenadas $(\mathrm{X}, \mathrm{Y})$ que definen, en el colorímetro, el color de las muestras de quesos de los diversos tratamientos, evaluados a través del período de maduración. Se observa que en el transcurso de ésta, ocurre un cambio continuo y permanente en las coordenadas que determinan el color, lo que demuestra la variación de color ocurrida en este período.

\section{FIGURA 3}

\section{Perfil de evaluación sensorial en queso Chanco madurado (*) con y sin suero}

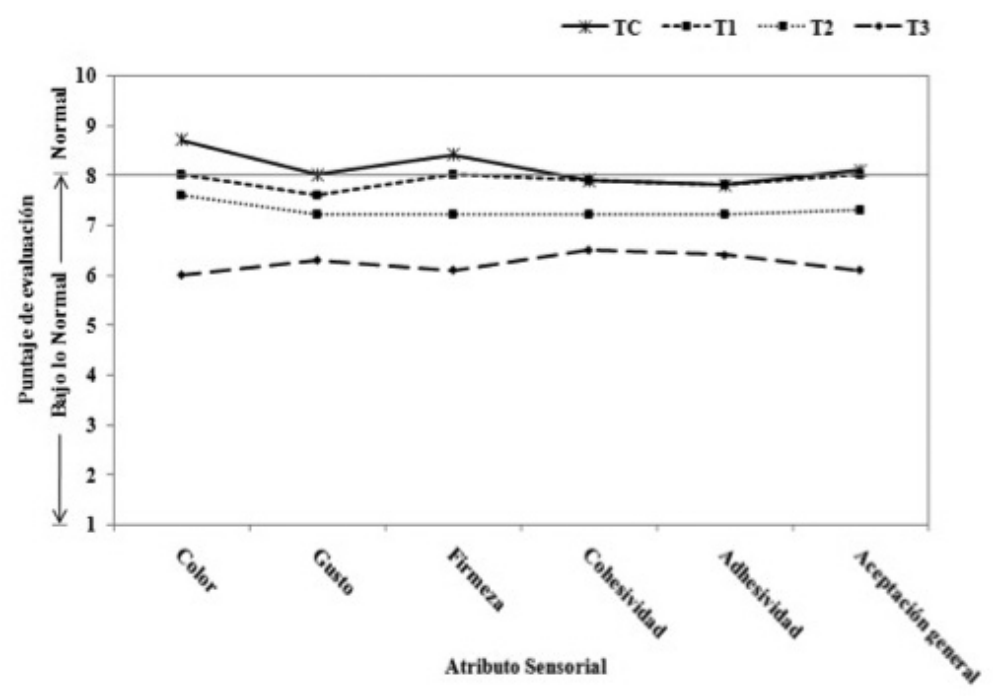


El coeficiente obtenido en la correlación de Spearman, es de 0,771 (0.05 de significancia), lo que indica una asociación positiva entre la medida instrumental y la capacidad sensorial para percibir las diferencias del color. Por lo tanto, se evidencia que cualquiera de dichos métodos podría utilizarse en el control de calidad de las producciones.

Aroma y gusto: El aroma del queso Chanco debe ser puro y medianamente intenso. En la evaluación del atributo olor, no hubo concordancia entre los valores asignados por los panelitas (Test de Kendall), aunque en general dieron un valor "normal" en el tratamiento control y "bajo lo normal" en los otros tratamientos. Los defectos más detectados por los jueces fueron "olor ácido", seguido de "olor penetrante".

El atributo "gusto" presentó concordancia entre panelistas y además diferencias estadísticas significativas entre el tratamiento control, T1 y T2 todos "normal" $(8,0 ; 7,6$ y 7,2) frente al T3 "bajo lo normal" con 6,3 a los 28 días de maduración, resultados semejantes a los obtenidos a los 42 días.

Los defectos encontrados se relacionan directamente con el comportamiento de la fermentación, cuya acidi- ficación fue lenta en el proceso pero persistió anormalmente, en forma continua durante la maduración. Un $\mathrm{pH}$ inusualmente bajo durante la maduración retarda la velocidad de la proteólisis, lo que afecta el desarrollo de la textura y sabor de los quesos. Además, es posible que por la presencia de proteínas séricas desnaturalizadas, específicamente la $\beta$-lactoglobulina, se inactiven algunas enzimas (plasmina) provocando retraso particularmente en la degradación de las caseínas, lo que no permitiría lograr el equilibrio de los componente presentes en la cuajada fresca y de los originados en la degradación enzimática de los constituyentes iniciales, durante la maduración normal del queso (33).

\section{Textura}

Respecto a la Firmeza, el queso Chanco debe tener una masa semiblanda y mantecosa; por lo tanto considera como defecto una masa muy firme o muy blanda $(19,37,39)$. Los resultados del test Kruskal-Wallis y la gráfica de Box y Whisker, denotan que existe diferencia estadísticamente significativas entre los tratamientos. A los 28 días del período de maduración de los quesos se presentan diferencias en los grupos TC-T2 y TC-T3,

\section{TABLA 2}

\section{Evolución del color (instrumental) en la maduración del queso Chanco $(*)$}

Tratamiento $\begin{gathered}\text { Días de } \\ \text { maduración }\end{gathered}$

C

0

14

28

42

2

\section{0}

14

28

42

3

\section{Valores de coordenadas (Colorímetro) \\ $\mathbf{X}$}

0,41 a

0,44 a

0,45 a

$0,44 \mathrm{a}$

0,42 a

0,43 a

$0,43 \mathrm{~b}$

$0,43 a b$

0,42 a

0,41 a

$0,44 \mathrm{~b}$

$0,44 \mathrm{~b}$

0,41 a

0,42 a

$0,40 \mathrm{~b}$

$0,40 \mathrm{~b}$
$0,37 \mathrm{a}$

0,38 a

0,41 a

$0,40 \mathrm{a}$

$0,37 \mathrm{~b}$

$0,37 \mathrm{a}$

$0,40 \mathrm{a}$

$0,40 \mathrm{a}$

$0,38 \mathrm{~b}$

0,38 a

0,40 a

0,38 a

$0,35 \mathrm{~b}$

0,39 a

0,39 a

0,38 a

\section{Color según esquema} sistema CIE

\author{
Blanco Naranjo \\ Naranjo
}

Naranjo Amarillento

Naranjo Amarillento

Naranjo Rosa

Naranjo Rosa

Naranjo Amarillento

Naranjo Amarillento

Blanco Naranjo

Blanco Naranjo

Naranjo Amarillento

Naranjo

Naranjo Rosa

Naranjo

Blanco Naranjo

Blanco Naranjo

(*) Promedio de las tres repeticiones

Letras distintas indican diferencia significativa entre tratamientos, a un nivel del $95 \%$ de confianza. 
resultando $\mathrm{T} 1$ el único tratamiento estadísticamente igual al control, con valores 8,4 (TC) y para T1 de 8,0 lo que los ubica en el rango "normal", los tratamientos 2 y 3 con 7,2 y 6,2, respectivamente, resultaron con masa "excesivamente blanda".

El Chanco es un queso bastante cohesivo, es decir, debe deshacerse homogéneamente en la boca, se considera defecto una masa desmenuzable, heterogénea e irregular al paladar $(37,41)$

A los 28 días de maduración, el máximo valor en cohesividad fue de 7.9, para TC y T1; correspondiente a "normal", tampoco hubo diferencia significativa con T2 $(7,2)$, todos diferentes a T3 que presentó 6,5; lo que lo ubica en el concepto de "bajo lo normal", al presentar una masa "desmenuzable". La cohesividad obedece principalmente a los tipos de enlaces que se presenten entre las micelas de las caseínas, al nivel de proteólisis y al contenido de grasa del producto, por lo que al parecer algunos de estos últimos aspectos se vieron afectados por el agregado de suero.

El queso Chanco se debe pegar ligeramente al paladar para a su vez rápidamente despegarse, lo que corresponde a una "mediana" adhesividad (37, 40, 41). A los 28 días de maduración los puntajes asignados para los tratamientos control, 1 y 2 se observan sin diferencias significativas con valores de 7,8 para los dos primeros 7,2 para T2. T3 resultó diferente con 6,4; lo que ubica este tratamiento en el concepto de "bajo lo normal", por lo que los jueces reportaron percibir un queso mas "pegajoso" que lo normal.

Aceptación general: En la figura 3, se visualiza que el rango donde se encuentran los puntajes totales asignados por los jueces a los 28 días de maduración, oscila entre 8,1 y 6,1 donde el tratamiento control presentó el máximo puntaje, siendo estadísticamente igual a T1 $(8,0)$, estadísticamente diferente a T2 $(7,3)$ y también de ellos con el tratamiento 3 ( $6 \%$ de suero) que presentó el mínimo (6.1).

De acuerdo a los resultados obtenidos, el tratamiento 1 ( $2 \%$ de suero) presenta características sensoriales similares al tratamiento control, lo que confirma el potencial uso, en la elaboración de esta variedad de queso, del suero en polvo, producto lácteo barato y valioso en términos nutritivo dietéticos.

\section{RESUMEN}

Se evaluó la influencia ejercida por el agregado de proteínas séricas, sobre los cambios bioquímicos ocurridos en la maduración del Chanco. Se plantearon cuatro tratamientos: TC (control), y T1, T2 y T3 con agregado de $2,4,6 \%(\mathrm{~m} / \mathrm{m})$ de suero en polvo, respectivamente, usando un diseño factorial de 3 x 4 (4 tratamientos y 3 repeticiones). En el control de $\mathrm{pH}$, humedad, materia grasa, sólidos totales, proteína, cenizas y sal, a los 0 , 14, 28 y 42 días, se usaron métodos estandarizados, IDF-FIL, AOAC y Normas Chilenas. Color instrumental por el colorímetro Color Tester LFM 1, aw por el analizador LUF y por fórmula. En la evaluación sensorial se usó el test de Comparación Múltiple (4 atributos). Los resultados indican que en los tratamientos con suero la glicólisis se prolonga durante la maduración. Humedad, grasas y cenizas se incrementan en T3 y T4 y la actividad de agua (aw) desciende más lentamente en esos quesos. En el perfil textural resultan estadísticamente iguales el Control y T1. Se concluye que la adición de suero al proceso de Queso Chanco es factible pero en niveles limitados.

Palabras clave: Queso Chanco, suero lácteo en polvo.

Dirigir la correspondencia a:

Profesora

Carmen Brito C

Instituto de Ciencia y Tecnología de los Alimentos Universidad Austral de Chile.

Valdivia

Chile.

Fono: (56 - 63) 221250

Fax: $(56-63) 221355$.

E mail: cbrito@uach.cl.

Agradecimientos: A la Dirección de Investigación (DID) de la Universidad Austral de Chile y a la Cooperativa Agrícola y Lechera CAFRA Ltda, por el apoyo en el financiamiento de este estudio.

\section{BIBLIOGRAFÍA}

1. Centro de Producción más limpia de Nicaragua. CPML-N. Estudio de pre-factibilidad para la instalación de una planta procesadora de bebidas para infantes a base de lactosuero, 2004. http://www. cpmlnic.org.ni/infotec/pml.htm.

2. Foegeding E. \& Luck P. Roginski, H. Fuquay, J. Fox, P. (edits). Encyclopedia of Dairy Sciences. Academia Press. London. 2005; 3: 1957-1960.

3. Stone N. y Kuhner R. Efectos de la modificación en la dieta en el tratamiento de la obesidad. Énfasis en la mejoría de los resultados vasculares. En: Robinson K. Clínicas Médicas de Norteamérica. Modificaciones de factores de riesgo en cardiopatías. Ed. 
Mc Graw-Hill Interamericana. México. 2000.

4. Zemel M. Mechanisms of dairy modulation of adiposity. Symposium: Dairy product components and weight regulation. Am Soc Nutr Sci 2003. 252S-256S.

5. Teegarden D. Calcium intake and reduction in weight or fat mass. Symposium: Dairy product components and weight regulation. Am Soc Nutr Sci 2003. 249S-251S

6. Shamik J. and Yanovski J. Calcium Intake and Adiposity. Review Article. Am J Clin Nutr 2003; 77: 281-287.

7. Muzzo S. Evolución de los problemas nutricionales en el mundo. El caso de Chile. Rev Chil Nutr 2002; 29: 78-85.

8. Vásquez F, Salazar G, Andrade M, Díaz E, Rojas J. Ingesta alimentaria de preescolares obesos asistentes a los jardines infantiles de la JUNJI. Rev Chil Nutr 2004; 31 (2):100-108.

9. Vio F. y Salinas J. Promoción de salud y calidad de vida en Chile: una política con nuevos desafíos. Rev Chil Nutr 2006; 33 (supl. 1): 252-259.

10. Chile. Ministerio de Salud. Intervención Nutricional a través del ciclo Vital para la Prevención de Obesidad y otras Enfermedades Crónicas No Transmisibles. Encuesta Nacional de Salud. Consejo Ejecutivo $113^{\mathrm{a}}$ reunión 27 de noviembre, 2003.

11. Mendoza C, Pinheiro A. C, Amigo H. Evolución de la situación Alimentaria en Chile. Rev Chil Nutr 2007; 34 (1): 62-70.

12. Fox PF. Milk proteins as food ingredients. Int $\mathbf{J}$ Dairy Tech 2001; 54(2)41-55

13. Candioti MC, Zalazar CA, Meinardi CA, Hynes E. Susceptibility of whey proteins to the action of commercial proteases used in food processing. Australian J of Dairy Tech 2001; 56(1):35-37.

14. Balagdatas, JV, Hutchinson FM, Krochta JM, Sumner, DA. Anticipating market effects of new uses for whey and evaluating returns to research and development. J Dairy Sci 2003; 86:1662 - 1672.

15. Chile. Ministerio de Agricultura. Oficina de Estudio y Políticas Agrarias. ODEPA. Boletín de la Leche 2007. Santiago. 2008.

16. Jameson G. \& Lelievre J. Effects of whey proteins on cheese characteristics. IDF-FIL. Bulletin 1996; 313: 3-8.

17. Steffl A, Hafenmair M, Hechler A, Hinrichs J. Influence of whey protein particles on the renneting properties of milk. Milchwissenschaft 1999; 54(9): 510-513.

18. Kjaergaard J. \& Stapelfeldt H. Incorporation of whey proteins in cheese. Including the use of ultra- filtration. IN: Factors affecting the yield of cheese. IDF especial issue: 1991; 9301, 88-108.

19. Chile. Instituto Nacional de Normalización, INN. Productos lácteos, queso Chanco. Requisitos. Norma Chilena 2090. 1999.

20. Oliveira M \& Brito C. A Y Tamime (Edit) Brined cheeses. Society of Dairy Technology Series. London. Blackwell Publishing. 2006, pp. 211 - 248.

21. Chile. Instituto Nacional de Normalización, INN. Leche y productos lácteos. Determinación de pH. Norma Chilena 1671. 1999.

22. Pinto, M; S Vega y N Perez. Métodos de análisis de la leche y derivados. Garantía de Calidad. Uniprint. Imprenta Universitaria SA. Valdivia. Chile. 1998.

23. IDF, International Dairy Federation. Cheese and processed cheese. Determination of the total solids content (Reference method) i.s. IDF - FIL. 4-A. Int. Dairy Fed., Brussels, Belgium. 1982.

24. IDF, International Dairy Federation. Milk. Determination of nitrogen content. (Reference method) i.s. IDF - FIL. 20B. Int. Dairy Fed., Brussels, Belgium. 1993.

25. Association Official analysis Chemical. AOAC. Determinación de cenizas: Método gravimétrico. Official Method 935.42. 15th Ed. Washington. 1990.

26. Association Official analysis Chemical. AOAC. Determinación de Cloruros $(\mathrm{NaCl})$. Método por titulación colorimétrica. AOAC. Official Method 935.43. 15th Ed. Washinton. 1990.

27. Lange D. Operating instructions Guide. Color Tester LFM 1. Edition 02. BDA 117. P. irreg. s/f.

28. Rodel W. \& Leistenr L. Simple aw-value analyzer for the practice. Die Fleischwirtschaft 1971; 12: 1800-1802.

29. Esteban M \& Marcos A. Equations for calculation of Water activity in cheese from its Chemical composition: A Review. Food Chem 1990; 35 (3):179-186

30. Scott R. Fabricación de queso. Acribia S.A, Zaragoza. 1991.

31. Brito C, Morales O, Molina L.H, Pezzot R. y Pinto M. Evolución de diversos parámetros de maduración de Queso Chanco tipo campo almacenado a altas temperaturas. I. Parámetros fisicos y químicos. Agrosur 1995; 23 (2): 95 - 106.

32. Clarck D. Fat replacers and fat subtitutes. Food Tech 1994. 48(12): 86.

33. Eck A. El queso. Ed. Omega S.A, Barcelona. 1990.

34. Asmundson R, Liuj S, Gopal P, Holland R, Crow V. Influence of Reduced Water Activity on Lactose Metabolism by Lactococcus lactis subsp. cremoris 
at Differerent pH values. Applied J Dairy Sci 1984; 67:2723-2733.

35. Fox PF, McSweeney PLH, Cogan TM \& Guinee TP. (Edits). Cheese. Chemistry, Physics and Microbiology. 3th ed. V1 and V2. Elsevier Academic Press. London. 2004.

36. Punidadas P, Tung M, y Feirtag J. Potencial use of homogenized whey protein dispersions and process modification for the manufacture of low fat and reduced fat cheddar type cheese. Int J Dairy Tech 2000; 53 (2): 45-50.

37. Brito C, Morales O, Molina LH, Pezzot R. y Pinto M. Evolución de diversos parámetros de maduración de Queso Chanco tipo campo almacenado a altas temperaturas. II. Proteólisis. Agrosur 1996;
24 (1): $1-13$.

38. Roupas, P. y Mead, D. Whey protein s and pizza cheese. Dairy Industry Int 2001; 66(9):16-18.

39. Molina LH, Barría M, Brito C. Características de calidad química y sensorial del queso Chanco de Campo del Mercado en Chile. Alimentos 1996; 21(1-2): 25-37.

40. Hort J, Greys G, Woodman J. Changes in the perceived textural properties of Cheddar cheese during maturation. J Sensory Stu 1997; 12 (4): $255-266$.

41. Brito C, Astete MA, Pinto M, Molina LH. Maribo cheese manufactured with concentrated milk: characteristics, maturation and yield. Int J Dairy Tech 2000; 53(1):6-12. 